\title{
Elimination of daily routine chest radiographs does not change on-demand radiography practice in post-cardiothoracic surgery patients
}

Onno Mets, ${ }^{a}$ Peter E. Spronk, MD, PhD, ${ }^{\text {b,c }}$ Jan Binnekade, PhD, ${ }^{\mathrm{a}, \mathrm{d}}$ Jaap Stoker, MD, PhD,

Bas A. J. M. de Mol, MD, PhD, ${ }^{f}$ and Marcus J. Schultz, MD, PhD ${ }^{a, c, g}$

From the Departments of Intensive Care Medicine, ${ }^{\text {a }}$ Clinical Epidemiology and Biostatistics, ${ }^{\mathrm{d}}$ Radiology, ${ }^{\mathrm{e}}$ and Cardiopulmonary Surgery, ${ }^{\mathrm{f}}$ and the Laboratory of Experimental Intensive Care and Anesthesiology (L.E.I.C.A.), ${ }^{\mathrm{g}}$ Academic Medical Center, University of Amsterdam, Amsterdam, the Netherlands; the Department of Intensive Care Medicine, ${ }^{\mathrm{b}}$ Gelre Hospital (Location Lukas), Apeldoorn, the Netherlands; and HERMES Critical Care Group, ${ }^{\mathrm{c}}$ Amsterdam, The Netherlands.

Received for publication Nov 16, 2006; revisions received Jan 11, 2007; accepted for publication Feb 5, 2007.

Address for reprints: Marcus J. Schultz, $\mathrm{MD}, \mathrm{PhD}$, Department of Intensive Care Medicine, Mail stop C3-329, Academic Medical Center, Meibergdreef 9, 1105 AZ Amsterdam, The Netherlands (E-mail: m.j. schultz@amc.uva.nl).

J Thorac Cardiovasc Surg 2007;134:139-44 $0022-5223 / \$ 32.00$

Copyright @ 2007 by The American Association for Thoracic Surgery

doi:10.1016/j.jtcvs.2007.02.029
Objective: We sought to determine the effect of elimination of daily routine chest radiographs on chest radiographic practice in cardiothoracic surgery patients in the intensive care unit and the post-intensive care unit ward.

Methods: We used a prospective, comparative study design with an intervention in a 28-bed intensive care unit/post-intensive care unit ward (including a 4-bed medium-care unit) in a university hospital. Cardiothoracic surgery patients were admitted to the intensive care unit during a period of 6 months ( 3 months before intervention and 3 months after intervention). Daily routine chest radiographs in the intensive care unit were eliminated; all chest radiographs required a clinical indication. Routine chest radiographs were not performed in the post-intensive care unit ward, both before and after the intervention.

Results: Before intervention, in the intensive care unit 353 daily routine chest radiographs and 261 on-demand chest radiographs were obtained in 175 patients; after intervention, 275 on-demand chest radiographs were obtained in 163 patients. Before intervention, in the post-intensive care unit ward 413 on-demand chest radiographs were obtained in 167 patients; after intervention, 445 on-demand chest radiographs were obtained in 161 patients. In the intensive care unit the number of chest radiographs per patient day decreased from $1.8 \pm 0.6$ to $1.1 \pm 0.6$. In the post-intensive care unit ward the number of chest radiographs per patient per day was $0.4 \pm 0.2$, both before and after the intervention. Slightly more unexpected abnormalities were found in the on-demand chest radiographs after the intervention. No negative influence on chest radiography timing, length of stay in the intensive care unit and hospital, and readmission rate was seen.

Conclusions: Elimination of daily routine chest radiographs led to a decrease of the total number of chest radiographs obtained per patient per day in the intensive care unit and did not change chest radiography practice in the post-intensive care unit ward.

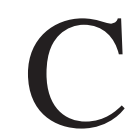

hest radiography is the most frequently performed radiology procedure in intensive care units (ICUs). ${ }^{1}$ Commonly, chest radiography is performed routinely as a complement to physical examination of critically ill patients $^{1,2}$; such radiographs are taken without any specific reason (so-called daily routine chest radiographs). Another strategy is the use of chest radiographs on indication only; such radiographs are ordered after a change in clinical status or change of supportive devices (so-called on-demand chest radiographs). The consensus opinion of the American College of Radiology expert panel is that daily routine chest radiographs are indicated in patients with acute cardiopulmonary problems and patients receiving mechanical ventilation (http://www.acr.org/ [last 


\section{Abbreviations and Acronyms \\ $\mathrm{CI}=$ confidence interval \\ ICU = intensive care unit \\ LOS $=$ length of stay}

assessed August 2006]). This guideline is followed for many ICU patients, including those after cardiothoracic surgery, at least in The Netherlands. ${ }^{2}$

A routine chest radiograph strategy seems debatable in ICU patients because the chest radiograph has only moderate accuracy in visualizing opacifications caused by cardiopulmonary abnormalities, and chest radiographs can be quite nonspecific as to cause. ${ }^{3}$ Moreover, radiographic abnormalities are dependent on ventilator settings at the time the chest radiograph is taken. ${ }^{4}$ Until now, a restrictive strategy has been compared with a daily routine strategy in only 2 clinical trials: in these studies the investigators showed that abandoning daily routine chest radiographs did not influence mortality and ICU length of stay (LOS). ${ }^{5,6}$

Recently, we showed that daily routine chest radiographs seldom reveal unexpected clinically relevant abnormalities, and they rarely cause further action. ${ }^{7}$ In view of these findings, we abandoned daily routine chest radiographs for all patients in our ICU, including those after cardiopulmonary surgery. It was feared that the abandoning of daily routine chest radiographs would result in a sharp increase of on-demand chest radiographs; in addition, it was speculated that many abnormalities would be missed, resulting in an increase of diagnostic value of the latter radiology studies. In the present study we determined the effect of the abovementioned change on chest radiography practice in the ICU and the post-ICU ward. In addition, we compared the diagnostic efficacy of chest radiographs, ICU and hospital LOS, and readmission rates to the ICU before and after the intervention.

\section{Material and Methods \\ Study Design}

We designed a prospective comparative study to investigate the effect of abandoning daily routine chest radiographs. The study protocol was approved by the ethics committee of the Academic Medical Center of the University of Amsterdam. The requirement to obtain informed consent was waived because this study evaluated 2 commonly applied chest radiography strategies.

\section{Setting}

The study took place in the 28-bed ICU of the Academic Medical Center, a university hospital in The Netherlands. The patient population consists of adult cardiothoracic surgery patients. The ICU is a "closed format" department in which patients are under the direct care of the ICU team, consisting of 8 full-time intensivists, 8 subspecialty fellows, 12 residents, and occasionally 1 intern.
The cardiothoracic post-ICU ward contains a 4-bed mediumcare unit that provides care for post-cardiothoracic surgery patients after discharge from the ICU; it is hospital policy to admit these patients to the medium-care unit of the cardiothoracic surgery ward for at least 24 hours, and thereafter, they are transferred to a normal bed in the cardiothoracic surgery ward when their condition is judged to be stable. The medical team on the post-ICU ward consists of 6 residents who are supervised by a daily (alternating) supervisor (one of the cardiothoracic surgeons).

\section{Patients}

Patients consisted of all post-cardiothoracic surgery patients admitted after surgical intervention to the ICU in a period of 3 months before intervention (phase 1) and 3 months directly after intervention (phase 2). Per day, 3 to 5 cardiothoracic surgery patients were admitted to the ICU. All cardiothoracic surgery patients are transferred to the ICU after the surgical procedure; after arrival in the ICU, a chest radiograph is obtained to ensure proper position of indwelling lines and the endotracheal tube and to determine the presence of pneumothorax or major atelectasis (these radiographs were considered to be on-demand radiographs). During phase 1 , daily routine radiographs were obtained between 8 and 9 AM each day.

\section{Procedure}

The intervention consisted of a change in the ordering practice of chest radiographs: only on-demand chest radiographs were to be obtained. The attending physicians were not blinded for this change in strategy; in fact, they were the ones who had to abandon the daily routine strategy. In addition, there were no standing orders for chest radiographs starting the new strategy. Each chest radiograph required a clinical indication, such as admittance to the ICU; insertion of central venous lines, intra-aortic balloon pump, or tracheal and chest tubes; an increase in oxygen requirement; or a change in pulmonary secretions with or without fever. Only if one of these indications was present and after consulting the supervisor (in case of residents/interns) was an on-demand chest radiograph obtained.

\section{Data Collection}

For all observed patients, data were collected on the type of cardiothoracic surgery, ICU and hospital LOS, and readmission to the ICU. For both study phases, the number and timing of chest radiographs obtained in the ICU were registered. Chest radiographic volume data and timing from the step-down facility were collected from day 1 (day of discharge from ICU) until day 7 or discharge, whatever came first.

\section{Diagnostic Value of Chest Radiographs}

For each chest radiograph obtained in the ICU, the subspecialty fellow, resident, or intern completed a specially developed data sheet, which was printed on the back of the normal radiology request form, as described previously. ${ }^{7}$ Clinically expected abnormalities were documented on this data sheet. The attending physician ticked several options to indicate whether a certain finding was expected and whether it was "old" (ie, already present on preceding chest radiographs) or "new." All chest radiographs were interpreted by an independent radiologist on the day the chest 
Table 1. (Expected) findings on daily routine chest radiographs*

\begin{tabular}{|c|c|}
\hline Abnormality & Specific comment \\
\hline "Severe" atelectasis & $\geq 2$ lobes \\
\hline Large infiltrates & $\geq 1$ lobe \\
\hline "Severe" pulmonary congestion & $\begin{array}{l}\text { More than usual postsurgical } \\
\text { changes }\end{array}$ \\
\hline "Severe" pleural effusion & $\begin{array}{l}\text { More than usual postsurgical } \\
\text { changes }\end{array}$ \\
\hline $\begin{array}{l}\text { Pneumothorax or } \\
\text { pneumomediastinum }\end{array}$ & Any abnormal air collection \\
\hline $\begin{array}{l}\text { Malposition of oropharyngeal } \\
\text { tube }\end{array}$ & $\begin{array}{l}<2 \mathrm{~cm} \text { from carina or above } \\
\text { stem cords }\end{array}$ \\
\hline Malposition of intravenous lines & $\begin{array}{l}\text { Tip in right atrium or outside } \\
\text { lumen (pulmonary artery } \\
\text { catheter: tip in right } \\
\text { atrium) or change in } \\
\text { position }\end{array}$ \\
\hline \multicolumn{2}{|l|}{$\begin{array}{l}\text { Malposition of intra-aortic } \\
\text { balloon pump }\end{array}$} \\
\hline Malposition of gastric tube & Tip outside of the stomach \\
\hline Malposition of drains & $\begin{array}{l}\text { Displacement }>5 \mathrm{~cm} \text { or } \\
\text { outside pleural space }\end{array}$ \\
\hline
\end{tabular}

Adapted and modified from Graat and colleagues. ${ }^{7}$ Abnormalities were scored by residents or clinical fellows if expected and separately by a radiologist if present.

radiograph was obtained. Similar to the ICU physicians, the radiologist structurally interpreted all chest radiographs as described previously (Table 1). If an abnormality was worsening, it was categorized as "new."

For all chest radiographs performed in the post-ICU ward, chest radiography request forms were scored retrospectively for expected abnormalities by the researcher. For this, scanned request forms were retrieved from the hospital information system. The same system was used to collect interpretation by radiologists. The following definitions were used: an expected finding was any new finding that had been predicted by the attending physician, and an unexpected finding was any new finding not predicted by the attending physician.

\section{Statistical Analysis}

Patient and clinical characteristics were summarized by using descriptive statistics. Chest radiograph volume is expressed as the mean $( \pm \mathrm{SD})$ number of chest radiographs per patient and chest radiographs per patient day. The samples from phase 1 and phase 2 and their differences are expressed as differences between proportions. If suitable, statistical uncertainties are expressed by $95 \%$ confidence intervals (95\% CIs). All calculations were performed with SPSS version 12.0.1 software (SPSS, Inc, Chicago, Ill).

\section{Results}

\section{Patients and Chest Radiographs}

We evaluated 338 patients over the 2 periods (patient demographic data are summarized in Table 2). For 6 patients, data from readmission were excluded. From patients in the ICU, 536 on-demand chest radiographs were obtained (261 from 169 patients in phase 1 and 275 from 163 patients in phase 2). In addition, 353 daily routine chest radiographs were obtained from 167 patients in phase 1 (in 2 patients no daily routine chest radiographs were obtained and daily routine chest radiographs were not obtained in phase 2). On the post-ICU ward, a total of 858 chest radiographs were obtained (413 from 167 patients in phase 1 and 445 from 161 patients in phase 2$)$.

\section{Volume Data Before and After Abandoning Daily Routine Chest Radiographs in the ICU}

In the ICU, the total number of chest radiographs per patient decreased after the intervention. In phase $13.5 \pm 4.3$ chest radiographs per patient were obtained, and in phase 2 this number decreased to $1.7 \pm 1.2$ (mean difference, $1.8 ; 95 \%$ CI, 1.1-2.5). On the post-ICU ward, the total number of chest radiographs per patient was $2.4 \pm 1.4$ and $2.8 \pm 1.4$ in phases 1 and 2, respectively (mean difference, $-0.4 ; 95 \%$ CI, -0.7 to -0.1$)$.

In the ICU after the intervention, the number of chest radiographs per patient per day decreased from $1.8 \pm 0.6$ to $1.1 \pm 0.6$ (mean difference, $0.7 ; 95 \% \mathrm{CI}, 0.6-0.8$ ). On the post-ICU ward, this number did not change.

\section{Table 2. Demographic data}

\begin{tabular}{|c|c|c|c|}
\hline & Phase $1(n=175)$ & Phase $2(n=163)$ & Difference $(95 \% \mathrm{Cl})$ \\
\hline Age (y), mean (SD) & $65.4(12.3)$ & $64.7(14.1)$ & $0.07(-2.1$ to 3.5$)$ \\
\hline Male sex (n [\%]) & $114(65.1)$ & $103(63.2)$ & $0.02(-0.08$ to 0.12$)$ \\
\hline ICU length of stay (d), mean (SD) & $2.5(4.5)$ & $1.8(1.5)$ & $0.7(-0.04$ to 1.42$)$ \\
\hline Length of day $1^{*}(d)$, mean (SD) & $0.47(0.10)$ & $0.48(0.11)$ & $-0.01(-0.03$ to 0.01$)$ \\
\hline \multicolumn{4}{|l|}{ Type of operation (n [\%]) } \\
\hline CABG & $88(50.3)$ & $68(41.7)$ & $0.09(-0.02$ to 0.19$)$ \\
\hline Valve replacement & $39(22.3)$ & $47(28.8)$ & $-0.07(-0.16$ to 0.03$)$ \\
\hline Combination of CABG + valve replacement & $27(15.4)$ & $22(13.5)$ & $0.02(-0.06$ to 0.10$)$ \\
\hline Othert & $21(12.0)$ & $26(16.0)$ & $-0.04(-0.12$ to 0.04$)$ \\
\hline
\end{tabular}

$C l$, Confidence interval; $S D$, standard deviation; $C A B G$, coronary artery bypass grafting. ${ }^{*}$ Day 1 is the first day in the post-intensive care unit ward until 12 AM. TValve plasty, patch plasty for patent foramen ovale, or procedures for congenital heart malformations. 

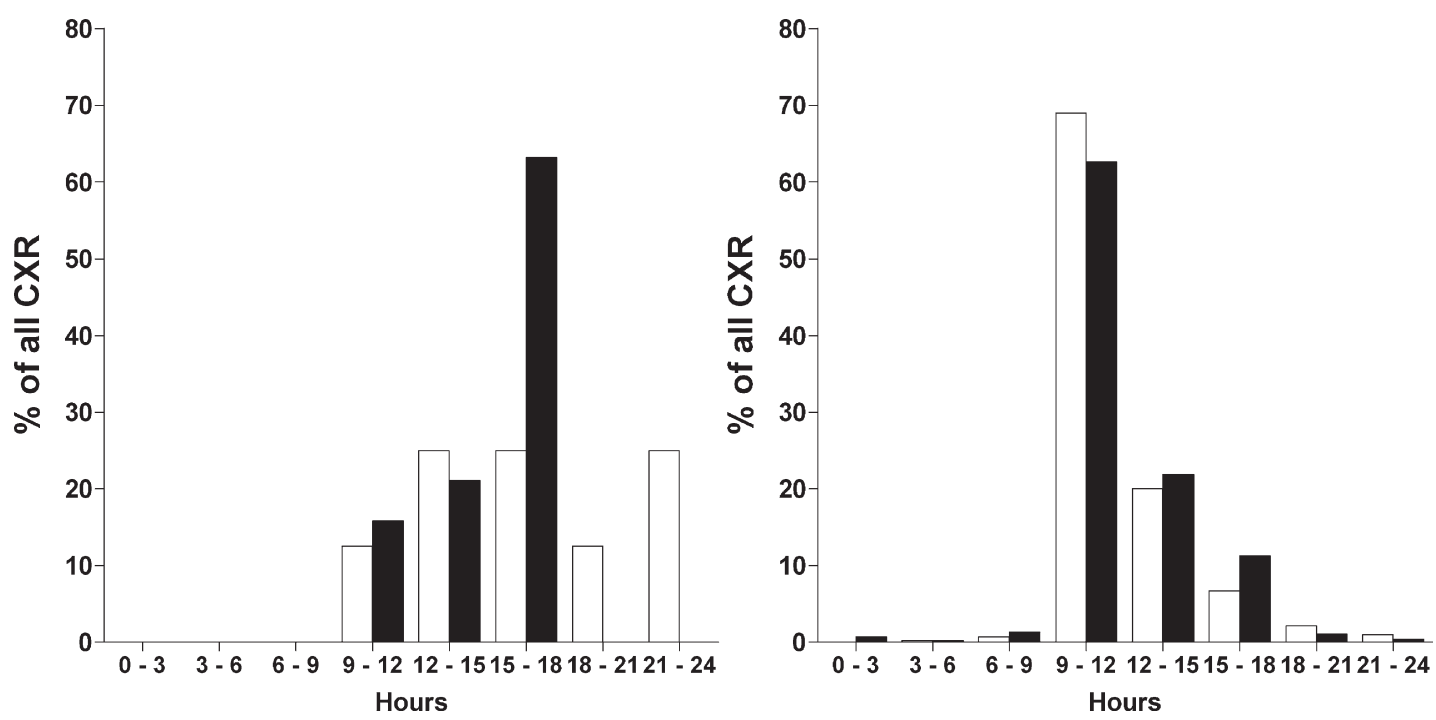

Figure 1. Left, Shift in distribution of chest radiographs taken on the day of transfer to the post-intensive care unit ward (open bars, phase 1; filled bars, phase 2). The number of chest radiographs performed on the day of transfer increased, although insignificantly, from 8 to 19 for the 3 months in phase 1 and phase 2, respectively. Right, The distribution of chest radiographs taken during the first 7 days after discharge from the intensive care unit was not different between the 2 phases of the study. CXR, Chest radiograph.

There were few changes in distribution of the timing of chest radiographs in the total period. Figure 1 shows a shift in distribution of chest radiographs taken on the day of transfer only (ie, the first day on the post-ICU ward) and the complete period after discharge from the ICU. It shows that the chest radiographs on the day of transfer in phase 2 were all performed during daytime and no longer in the evening or night. The number of chest radiographs performed on the day of transfer increased, although insignificantly, from 8 to 19 for the 3 months in phase 1 and phase 2, respectively.

Analysis of the number of chest radiographs showed no clear pattern in correlation with the type of surgical intervention.

\section{Diagnostic Value of Chest Radiographs}

In phase 1, 21 daily routine chest radiographs on the ICU demonstrated 24 new and unexpected abnormalities (5.9\% of all chest radiographs in $10.8 \%$ of the patients, Table 3 ). On-demand chest radiographs in the same phase revealed 32 new and unexpected abnormalities (12.0\% of all chest radiographs in $16.4 \%$ of the patients). Furthermore, none of the abnormalities expected by the attending physician were confirmed by the radiologist (Table 3). Twenty-one chest radiographs obtained in the post-ICU ward revealed 24 new and unexpected abnormalities (7.0\% of all on-demand chest radiographs in $24.7 \%$ of the patients). Only 8 of the expected abnormalities were confirmed to be present by the radiologist $(1.7 \%$ of all on-demand chest radiographs in $4.2 \%$ of the patients, Table 3 ).
In phase 2, 48 new and unexpected abnormalities were found by the radiologists $(18.7 \%$ of all on-demand chest radiographs in $29.3 \%$ of the patients, Table 3 ). On-demand chest radiographs obtained in the ICU showed only 3 new and expected abnormalities (1.1\% of all on-demand chest radiographs in $1.2 \%$ of the patients). On the chest radiographs obtained in the post-ICU ward, only 5 new and expected abnormalities were confirmed (1.1\% of all ondemand chest radiographs in $3.1 \%$ of the patients); 36 new and unexpected abnormalities were found $(9.5 \%$ of all on-demand chest radiographs in $40.3 \%$ of the patients, Table 4).

In addition to the significant increase in the number of new and unexpected abnormalities, there was a change in request behavior by the attending physicians in the ICU. In phase 2 physicians expected more new abnormalities, as noted on the request form. In the post-ICU ward, a similar increase in new and unexpected abnormalities was found, but the physicians did not change their request behavior after the intervention.

Also, with regard to the diagnostic value of chest radiographs, analysis showed no clear pattern in correlation with the type of surgical intervention.

Effect on LOS in the ICU, LOS in the Hospital, and Readmission Rate

A slight but statistically insignificant decrease in ICU LOS was found when comparing phase 1 and 2 (mean difference, 
Table 3. Incidence of new abnormalities on 353 daily routine and 536 on-demand chest radiographs obtained in the intensive care unit

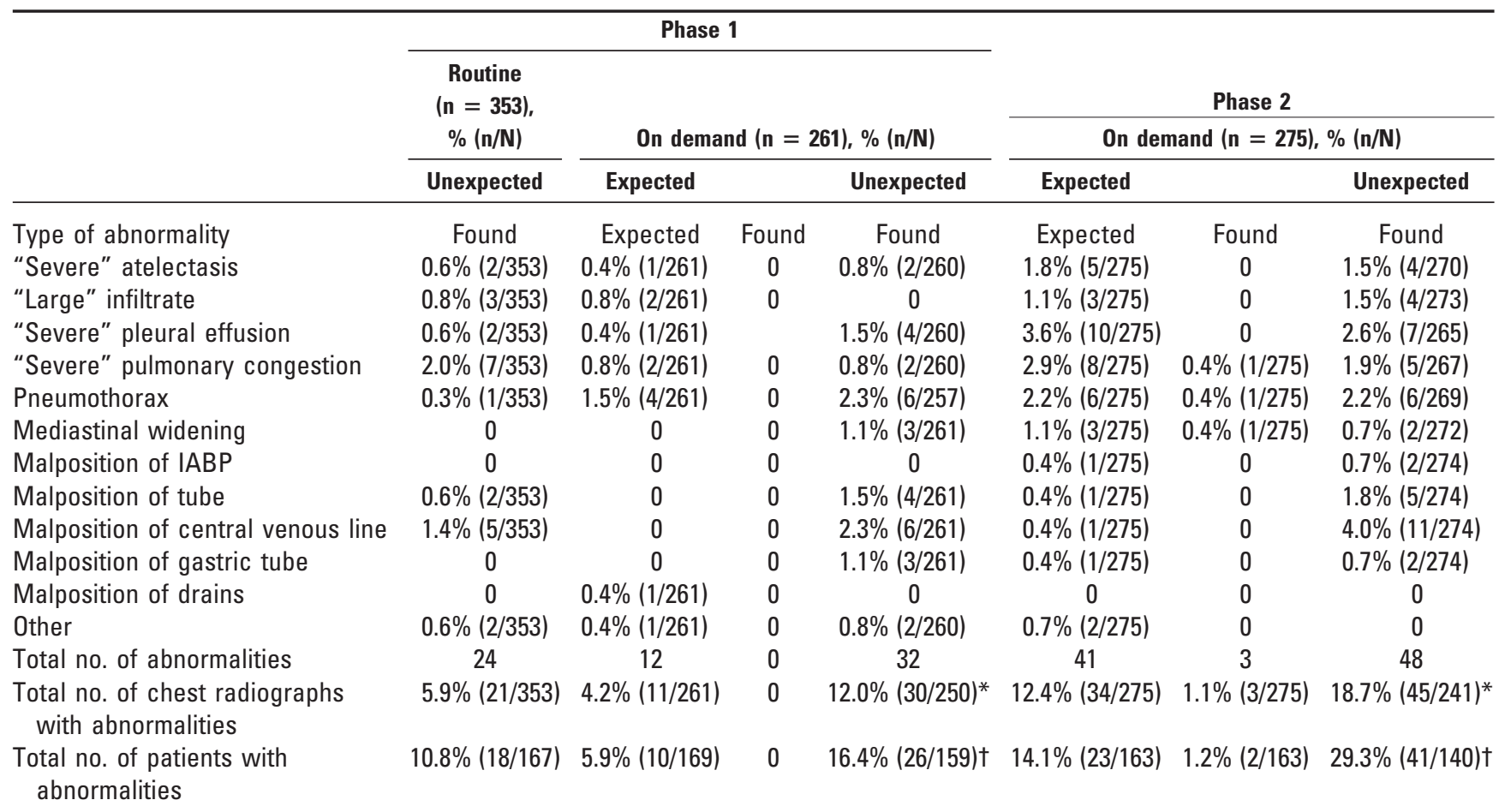

IABP, Intra-aortic balloon pump. *Number of chest radiographs without expected abnormalities. †Number of patients without expected abnormalities.

0.7 days; 95\% CI, 0.0-1.4 days); hospital LOS did not change (mean difference, -0.2 days; $95 \%$ CI. -1.7 to 1.3 days). The readmission rate was not different between the 2 phases (6\% vs $3 \%$; mean difference, $0.03 ; 95 \% \mathrm{CI},-0.0$ to 0.1$)$.

\section{Discussion}

The present study shows the outcome of elimination of daily routine chest radiographs in post-cardiothoracic surgery patients. The number of on-demand chest radiographs only slightly changed in the ICU or in the post-ICU ward. Al-

Table 4. Incidence of new abnormalities on 858 chest radiographs obtained in the post-intensive care unit ward

\begin{tabular}{|c|c|c|c|c|c|c|}
\hline \multirow[b]{3}{*}{ Type of abnormality } & \multicolumn{3}{|c|}{ Phase $1(n=413)$} & \multicolumn{3}{|c|}{ Phase $2(n=445)$} \\
\hline & \multicolumn{2}{|c|}{ Expected, \% (n/N) } & \multirow{2}{*}{$\begin{array}{c}\begin{array}{c}\text { Unexpected, } \\
\%(\mathrm{n} / \mathrm{N})\end{array} \\
\text { Found }\end{array}$} & \multicolumn{2}{|c|}{ Expected, \% (n/N) } & \multirow{2}{*}{$\begin{array}{c}\text { Unexpected, } \\
\%(\mathrm{n} / \mathrm{N})\end{array}$} \\
\hline & Expected & Found & & Expected & Found & \\
\hline "Severe" atelectasis & $19.6 \%(81 / 413)$ & $0.5 \%(2 / 413)$ & $0.6 \%(2 / 332)$ & $19.1 \%(85 / 445)$ & 0 & $0.8 \%(3 / 360)$ \\
\hline “Large" infiltrate & $20.1 \%(83 / 413)$ & $0.2 \%(1 / 413)$ & 0 & $20.4 \%(91 / 445)$ & $0.4 \%(2 / 445)$ & $1.1 \%(4 / 354)$ \\
\hline "Severe" pleural effusion & $20.1 \%(83 / 413)$ & $0.5 \%(2 / 413)$ & $2.4 \%(8 / 330)$ & $18.2 \%(81 / 445)$ & $0.4 \%(2 / 445)$ & $2.2 \%(8 / 364)$ \\
\hline "Severe" pulmonary congestion & $3.4 \%(14 / 413)$ & $0.2 \%(1 / 413)$ & $1.8 \%(7 / 399)$ & $2.7 \%(12 / 445)$ & 0 & $2.3 \%(10 / 433)$ \\
\hline Pneumothorax & $4.1 \%(17 / 413)$ & $0.5 \%(2 / 413)$ & $1.8 \%(7 / 396)$ & $3.1 \%(14 / 445)$ & $0.2 \%(2 / 445)$ & $2.1 \%(9 / 431)$ \\
\hline Malposition of central venous line & 0 & 0 & 0 & 0 & 0 & 0 \\
\hline Malposition of gastric tube & 0 & 0 & 0 & 0 & 0 & 0 \\
\hline Malposition of drains & 0 & 0 & 0 & 0 & 0 & $0.4 \%(2 / 445)$ \\
\hline Total no. of abnormalities & 278 & 8 & 24 & 283 & 5 & 36 \\
\hline $\begin{array}{l}\text { Total no. of chest radiographs } \\
\text { with abnormalities }\end{array}$ & $27.1 \%(112 / 413)$ & $1.7 \%(7 / 413)$ & $7.0 \%(21 / 301)^{*}$ & $24.0 \%(107 / 445)$ & $1.1 \%(5 / 445)$ & $9.5 \%(32 / 338)^{*}$ \\
\hline $\begin{array}{l}\text { Total no. of patients with } \\
\text { abnormalities }\end{array}$ & $49.1 \%(82 / 167)$ & $4.2 \%(7 / 167)$ & $24.7 \%(21 / 85) \dagger$ & $52.2 \%(84 / 161)$ & $3.1 \%(5 / 161)$ & $40.3 \%(31 / 77) \dagger$ \\
\hline
\end{tabular}

${ }^{*}$ Number of chest radiographs without expected abnormalities. †Number of patients without expected abnormalities. 
though on-demand chest radiographs revealed more unexpected new abnormalities, after the intervention, there were no changes in LOS in the ICU, LOS in the hospital, and readmission.

Our study has several important drawbacks that should be mentioned. First, request behavior by physicians might significantly influence our analysis of the diagnostic value of chest radiographs. Indeed, physicians were not very specific on most of the request forms: 3 abnormalities were by far the most frequently expected abnormalities (atelectasis, infiltrates, and effusion), suggesting that they were ticked together without real suspicion. However, because this behavior was encountered in both phases, we think it remains possible to compare the 2 phases.

Second, because of the design of our study, it was not possible to collect data concerning the true clinical relevance of the found abnormalities. The clinical value of found abnormalities on chest radiographs of ICU patients has been found to be much lower than the diagnostic value. ${ }^{7}$

Finally, it must be noted that the present results might not simply be translated to other centers. Differences in staff and patient populations might be of great influence.

Our data are important in the discussion on whether a daily routine strategy should be replaced by an on-demand strategy. In our hospital both physicians from the cardiothoracic surgery department and personnel from the radiology department feared an increase in the number of ondemand chest radiographs in the ICU and the post-ICU ward. In addition, it was argued that potentially important abnormalities were to be missed when abandoning daily routine chest radiographs. This was not found in the present study. Indeed, there was only a slight increase in the number of chest radiographs in the post-ICU ward, and although the diagnostic efficacy of chest radiographs increased, this seemed unimportant for patient care because ICU LOS, hospital LOS, and the readmission rate were similar in the 2 phases of the study.

Recently, we showed that the diagnostic value of daily routine chest radiographs in the ICU was low. ${ }^{7}$ Our results are in accordance with these findings. The on-demand chest radiographs in the ICU had a low diagnostic value as well. After the intervention, the proportion of on-demand chest radiographs showing an unexpected abnormality increased slightly. This might be explained by the fact that all unexpected abnormalities that were found on daily routine chest radiographs in phase 1 were now found in the on-demand chest radiographs in phase 2. A similar change was found for on-demand chest radiographs in the post-ICU ward. Because there were no negative changes in ICU LOS, hospital LOS, or the readmission rate, we consider this slight change in absolute number not alarming.

Our data are in line with those of others. Krivopal and colleagues $^{6}$ and Price and associates ${ }^{5}$ both compared a daily routine chest radiography strategy with a restrictive chest radiography strategy in the ICU. Krivopal and colleagues ${ }^{6}$ performed a randomized control trial to determine differences in diagnostic, therapeutic, and outcome efficacy between these 2 strategies. They found no negative effect on ICU or hospital LOS. Price and associates ${ }^{5}$ performed a nonrandomized controlled study on the financial effect of changing chest radiography strategy. After elimination of daily routine chest radiographs in a pediatric ICU, they found a decrease in the number of chest radiographs per patient and an accompanying cost saving, while not influencing LOS.

The question of whether cessation of daily routine chest radiographs leads to an increase in missed cardiothoracic pathology that would have been detected by use of daily routine chest radiographs is hard to address based on the methodology of our study. We can only address this question on those patients who actually received an on-demand chest radiograph at sometime in the postoperative period; there are no data on patients who failed to receive an on-demand radiograph postoperatively. However, this issue has been studied recently in another center. ${ }^{8}$ In this study, in which daily routine chest radiographs were only obtained in a blinded fashion (ie, the radiographs were not presented to ICU physicians), daily routine chest radiographs hardly ever showed important abnormalities, which is very similar to the present report and a previous study.

In conclusion, elimination of daily routine chest radiographs in post-cardiothoracic surgery patients in our ICU did not result in changes in ICU and hospital LOS, nor did the readmission rate change. The intervention did not result in any important changes in chest radiography performance in the ICU and the post-ICU ward.

\section{References}

1. Trotman-Dickenson B. Radiology in the intensive care unit (part I) J Intensive Care Med. 2003;18:198-210.

2. Graat ME, Hendrikse KA, Spronk PE, Korevaar JC, Stoker J, Schultz MJ. Chest radiography practice in critically ill patients: a postal survey in the Netherlands. BMC Med Imaging. 2006;6:8.

3. Rubenfeld GD, Caldwell E, Granton J, Hudson LD, Matthay MA. Interobserver variability in applying a radiographic definition for ARDS. Chest. 1999;116:1347-53.

4. Hall JB, White SR, Karrison T. Efficacy of daily routine chest radiographs in intubated, mechanically ventilated patients. Crit Care Med. 1991;19:689-93.

5. Price MB, Grant MJ, Welkie K. Financial impact of elimination of routine chest radiographs in a pediatric intensive care unit. Crit Care Med. 1999;27:1588-93

6. Krivopal M, Shlobin OA, Schwartzstein RM. Utility of daily routine portable chest radiographs in mechanically ventilated patients in the medical ICU. Chest. 2003;123:1607-14.

7. Graat ME, Choi G, Wolthuis EK, Korevaar JC, Spronk PE, Stoker, J et al. The clinical value of daily routine chest radiographs in a mixed medical-surgical intensive care unit is low. Crit Care. 2006;10:R11.

8. Hendrikse K, Gratama J, ten Hove W, Bendien K, Rommes H, Schultz MJ, et al. Value of routine daily chest radiography in a non-university ICU: results of a controlled prospective study. Critical Care. 2006; 10(suppl 1):398. 